\section{A Bridge Over a Hamiltonian Path}

\author{
Gary McGuire and \\ Fiacre Ó Cairbre
}

g very year on the 16th of October, a group of people assemble at Dunsink Observatory in Dublin, Ireland, and walk to a bridge over the Royal Canal.

They are the staff and students of the Department of Mathematics at the National University of Ireland, Maynooth, and they are commemorating the creation of the quatemions by the Irish mathematician, William Rowan Hamilton, on 16 October, 1843, at that bridge. The walk is about three miles long and passes through very pleasant countryside, on the outskirts of Dublin.

William Rowan Hamilton was born in Dublin on 4 August, 1805. He spent his youth in Trim, Co. Meath, where he was educated by his uncle, James Hamilton. Hamilton lived in a large house, which is now called St. Mary's Abbey and is beautifully situated beside the Yellow Steeple, on the banks of the Boyne, across from the spectacular ruins of Trim castle. The house also served as the local school which was run by his uncle. Hamilton was appointed Astronomer Royal of Ireland at the age of twenty-one, and thus became a Professor while still an undergraduate at Trinity College, Dublin. He lived at Dunsink Observatory from that point, and the mathematical tourist can visit the observatory and see interesting items related to Hamilton. He would frequently walk along the Royal Canal to the Royal Irish Academy (some possessions of Hamilton are kept at the Academy, including some notebooks and his Icosian Game). It was on one of these walks that he had his famous moment of insight. He later described the event to his son [1]:

An electric current seemed to close; and a spark flashed forth, the herald (as I foresaw, immediately) of many long years to come of definitely directed thought and work. . . Nor could I resist the impulse-unphilosophical as it may have been-to cut with a knife on a stone of Brougham Bridge as we passed it, the fundamental formula with the symbols $i, j, k$, namely $i^{2}=j^{2}=k^{2}=i j k=-1$.

All traces of Hamilton's carving are gone, but there is a plaque on the bridge commemorating the moment. The bridge is in the suburb of Cabra and is now called Broombridge. There is a train station there of the same name, and it is the second stop on the Arrow train route from Connolly Station, in Dublin, to Maynooth. The plaque was erected in 1958 by the Taoiseach (Irish prime minister), Eamon de Valera, who had a degree in mathematics and lectured at Maynooth for two years (1912-1914). De Valera himself scratched the famous quaternion formula on his cell wall in Kilmainham jail in Dublin while imprisoned there in 1916. One can visit the jail and take a very interesting guided tour, and see in the museum a copy of the formula written by de Valera. In 1943 the Republic of Ireland issued a commemorative stamp to celebrate the centenary of the birth of the quaternions.

The current Taoiseach can see a statue of Hamilton near his office. The statue is on the steps of Government Buildings in Merrion Street in Dublin, and dates back to the time when the buildings housed the College of Science. This area may be of additional interest to the tourist, for a few hundred yards from Hamilton's statue is a monument to another famous Irishman, Oscar Wilde. The latter statue is in Merrion Square opposite the former home of Oscar Wilde. When Hamilton first met Oscar Wilde's mother, Lady Wilde, she asked him to be Oscar's godfather. He declined, but later became a close friend of Lady Wilde, who was a prominent writer under the pseudonym 


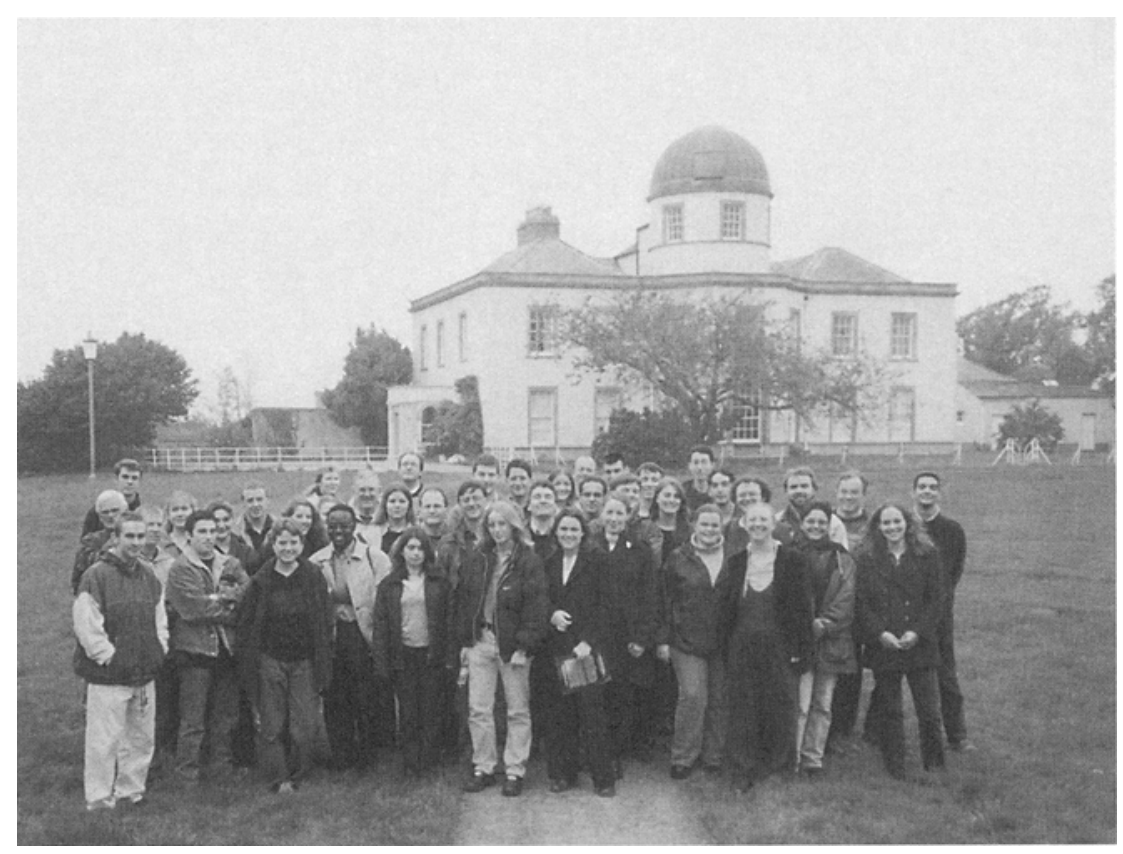

Figure 1. Staff and students of NUI, Maynooth, at Dunsink Observatory on 16 October, 1999.

Speranza. It is curious to note that Oscar Wilde was born on 16 October (1854). Who knows what Hamilton's reply might have been if he had known this!

A quaternion is an expression of the form $a+b i+c j+d k$ where $a, b, c, d$ are real numbers. Addition of quaternions is defined componentwise, and multiplication is defined using

$$
i^{2}=j^{2}=k^{2}=i j k=-1 .
$$

The multiplication is not commutative, i.e., $x y$ is not always equal to $y x$ when $x$ and $y$ are quaternions. In Hamilton's words: "the order of multiplication of these imaginaries is not indifferent." For example, one can show that $i j=$ $-j i=k$. Hamilton has been called the Liberator of Algebra because his quaternions shattered the convention that multiplication should be commutative in a number system. It is often said that he "freed algebra from arithmetic" because of this momentous development. His friend, the mathematician John Graves, said [1]:

I have not yet any clear view as to the extent to which we are at liberty arbitrarily to create imaginaries, and to endow them with supernatural properties. on the 2-sphere in 3-space. It is not hard to show that the transformation $x \mapsto q x \bar{q}$, where $x$ is pure and $q$ is a unit quaternion, is a rotation of 3-space. The correspondence between unit quaternions and rotations of 3-space is two-to-one.

One modern application of quaternions is that they frequently replace Euler angles in computer graphics for describing rotations of space. Thus, quaternions are now used, for example, in performing attitude determination in spacecraft like the space shuttle, and in computer games. The famous character Lara Croft in the computer game "Tomb Raider" was created [2] using quaternions!

Quaternions are nowadays of interest to algebraists in the form of general quaternion algebras over a field or ring $F$, where the coefficients $a, b, c, d$ come from $F$, and the defining relations are $i^{2}=\alpha, j^{2}=\beta, i j=k=-j i$, for some $\alpha$ and $\beta$ in $F$. The Hurwitz integral quaternions, where $a, b, c, d$ come from $\frac{1}{2} \mathbf{Z}$, are used in number theory and lattice constructions.

The quaternions played an important role in Maxwell's original formulation of electromagnetism. Vector analysis, which is indispensable in physics, is an offspring of quaternions. One can show that the unit quaternions

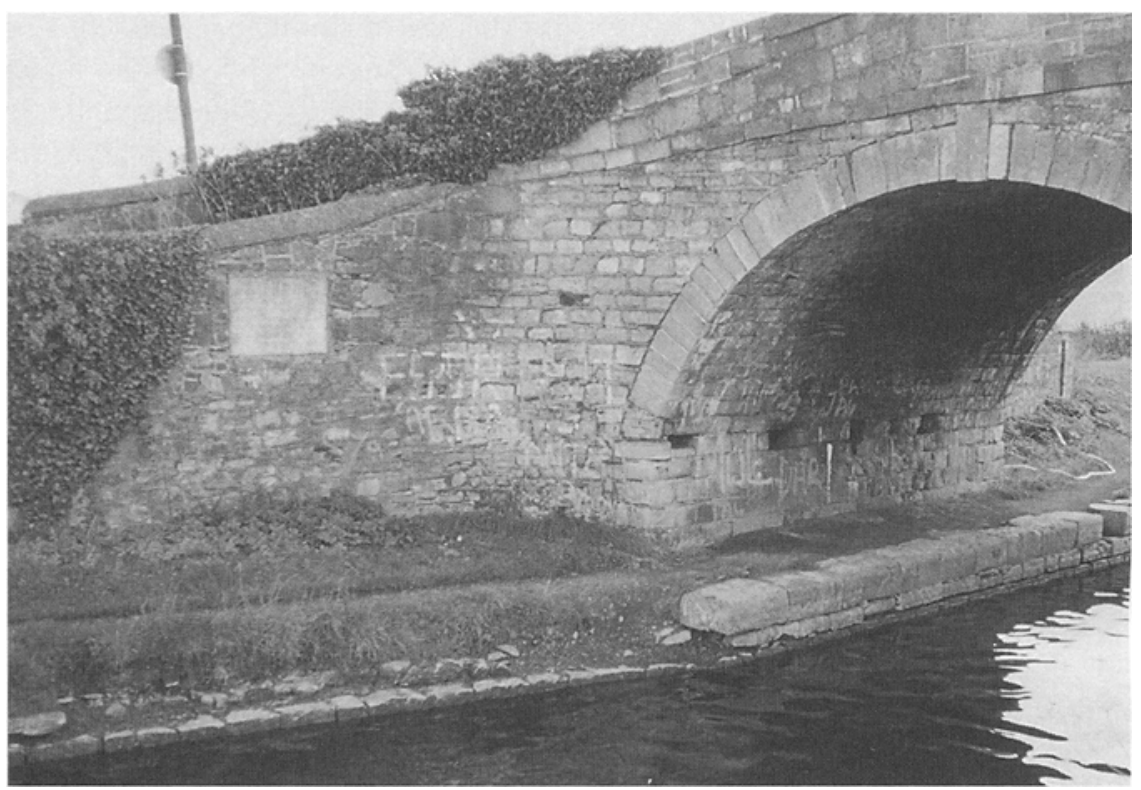

Figure 2. The bridge where Hamilton created the quaternions, with the plaque visible to the left of centre. 
are isomorphic to $S U(2)$, giving another viewpoint on this group which is of great importance in particle physics. Quaternions were generalised by Graves, and independently by Cayley, to the octonions which are currently under investigation for possible connections to particle physics. Hamilton made many other significant contributions to mathematics and physics, including his famous "Hamiltonian" which was essential for the development of Quantum Mechanics and is ubiquitous in physics.

Hamilton died at Dunsink on 2 September, 1865. In the same year, he had just become the first Foreign Associate to be elected to the newly established National Academy of Sci- ences of the United States of America. $\mathrm{He}$ is buried in Mount Jerome Cemetery in Dublin. For further information on Hamilton's life and works, see [1] and [3].

We conclude the article with an invitation and an open problem. We invite mathematical tourists to join us on the walk on 16 October every year. Contact either author for details, or see http://www.maths.may.ie. The open problem concerns Hamilton's eldest son, William Edwin, who emigrated to Canada. The last record of this son seems to be in Chatham, Ontario, where he worked for the Planet newspaper in 1891, and it is not known what became of him, and indeed if he married and had any children.

\section{REFERENCES}

[1] T. Hankins, Sir William Rowan Hamilton, Johns Hopkins University Press, Baltimore, 1980.

[2] N. Bobick, Rotating Objects Using Quaternions, 1998 (available online at http://www.gamasutra.com/features/programming/19980703/quaternions_01.htm).

[3] R. Dimitric and B. Goldsmith, Sir William Rowan Hamilton, Mathematical Intelligencer. 11, 2 (1989), 29-30.

\section{Department of Mathematics}

National University of Ireland

Maynooth

Co. Kildare

Ireland

e-mail: gmg@maths.may.ie

foc@maths.may.ie
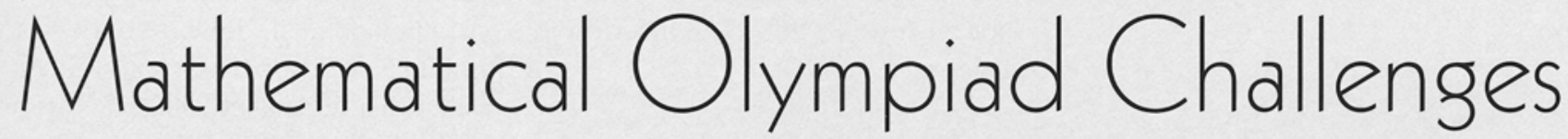

Titu Andreescu, American Mathematics Competitions, University of Nebraska, Lincoln, NE Răzvan Gelca, University of Michigan, Ann Arbor, MI

This is a comprehensive collection of problems written by two experienced and well-known mathematics educators and coaches of the U.S. International Mathematical Olympiad Team. Hundreds of beautiful, challenging, and instructive problems from decades of national and international competitions are presented, encouraging readers to move away from routine exercises and memorized algorithms toward creative solutions and non-standard problem-solving techniques.

The work is divided into problems clustered in self-contained sections with solutions provided separately. Along with background material, each section includes representative examples, beautiful diagrams, and lists of unconventional problems. Additionally, historical insights and asides are presented to stimulate further inquiry. The emphasis throughout is on stimulating readers to find ingenious and elegant solutions to problems with multiple approaches.

Aimed at motivated high school and beginning college students and instructors, this work can be used as a text for advanced problem-solving courses, for self-study, or as a resource for teachers and students training for mathematical competitions and for tcacher professional development, seminars, and workshops.

From the foreword by Mark Saul: "The book weaves together Olympiad problems with a common theme, so that insights become techniques, tricks become methods, and methods build to mastery... Much is demanded of the reader by way of effort and patience, but the investment is greatly repaid."

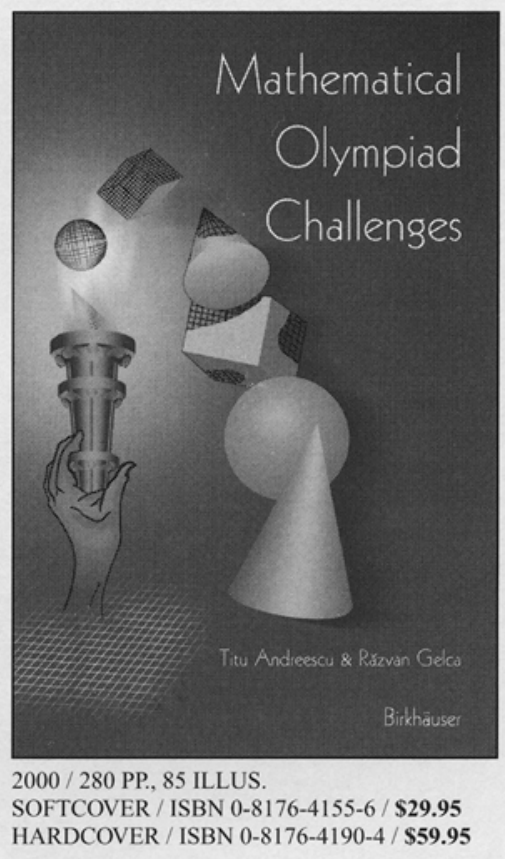

To Order: Call: 1-800-777-4643 • Fax: (201) 348-4505 • E-mail: orders@birkhauser.com • Visit: www.birkhauser.com Textbook evaluation copies available upon request, call ext. 669. Prices are valid in North America only and subject to change without notice. Birkhäuser Boston - International Publisher for the Mathematical Sciences 